\title{
Cell-to-cell variability in inducible Caspase9-mediated cell death
}

\author{
Yuan Yuan (D) ${ }^{1,2}$, Huixia Ren (D) ${ }^{1}$, Yanjun $\mathrm{Li}^{1}$, Shanshan Qin ${ }^{1,2}$, Xiaojing Yang $\mathbb{D}^{1 \times}$ and Chao Tang (D) ${ }^{1,3}$
}

(c) The Author(s) 2021

iCasp9 suicide gene has been widely used as a promising killing strategy in various cell therapies. However, different cells show significant heterogeneity in response to apoptosis inducer, posing challenges in clinical applications of killing strategy. The cause of the heterogeneity remains elusive so far. Here, by simultaneously monitoring the dynamics of iCasp9 dimerization, Caspase3 activation, and cell fate in single cells, we found that the heterogeneity was mainly due to cell-to-cell variability in initial iCasp9 expression and XIAP/Caspase3 ratio. Moreover, multiple-round drugging cannot increase the killing efficiency. Instead, it will place selective pressure on protein levels, especially on the level of initial iCasp9, leading to drug resistance. We further show this resistance can be largely eliminated by combinatorial drugging with XIAP inhibitor at the end, but not at the beginning, of the multiple-round treatments. Our results unveil the source of cell fate heterogeneity and drug resistance in iCasp9-mediated cell death, which may enlighten better therapeutic strategies for optimized killing.

Cell Death and Disease (2022)13:34 ; https://doi.org/10.1038/s41419-021-04468-z

\section{INTRODUCTION}

Inducible Caspase9 (iCasp9) is a cellular suicide gene that allows conditional cell elimination [1]. It comprises a human Caspase9 fused with an inducer-binding domain which could be dimerized by the Chemical Inducer of Dimerization (CID), AP20187, or AP1903 [2, 3]. iCasp9 is dimerized and activated by induced proximity of the inducer-binding domain, leading to activation of the apoptosis pathway [4]. Due to the efficient induction of cell death, iCasp9 has been used as one of the most promising killing strategies in cancer therapies [5-7] and adoptive cell therapies [8-14]. However, cells, especially cancer cells, cannot be eliminated $100 \%$ even treated with an extremely high dose of the drug. The incomplete killing of cells leads to drug resistance and hampers the further use of the iCasp9 suicide gene $[15,16]$. Consequently, one potential way to improve the performance of iCasp9 is through increasing drug efficacy. An understanding of the source of heterogeneous cell responses to the drug and how such heterogeneity contributes to resistance could lead to more effective treatment strategies.

Previous investigations into isogenic populations of tumor cells in response to apoptosis-inducing drugs revealed that the heterogeneity could emerge from non-genetic mechanisms, often through stochastic fluctuations in protein expression [17-20] or differences in dynamics [21, 22]. These studies mainly focused on the upstream of the apoptosis pathway. However, in the iCasp9 system, CID triggers the very downstream apoptosis pathway, so the source of heterogeneous response can be different. As depicted in Fig. 1a, in response to CID, iCasp9 is first dimerized and activated through the proximity of $\mathrm{DmrB}$ protein. Then, active iCasp9 triggers the activation of the executor Caspase3, which cleaves thousands of substrates in cells [23], resulting in irreversible cell death $[24,25]$. Opposing the proapoptotic iCasp9 and Caspase3, the antiapoptotic protein XIAP inhibits and degrades pro-apoptotic caspases once they are activated [26]. Thus, cell death is regulated by interactions between pro-apoptotic and antiapoptotic proteins.

In this work, we investigated potential causes for the heterogeneous cell response to inducer AP20187. We tagged iCasp9 with an mCherry fluorescence protein to track the dynamics of iCasp9 dimerization and incorporated a fluorescent substrate for Caspase 3 to report Caspase 3 activation and cell fate. We found that heterogeneous cell fates were originated from cell-to-cell variability in the initial iCasp9 expression and the ratio between XIAP and Caspase 3 expression levels (XIAP/C3 ratio). Moreover, we also observed significant heterogenous behaviors within surviving or dead cells, which were characterized by different dynamics of iCasp9 dimerization and Caspase 3 activation within the surviving cell population, and broad distribution of death time within the dead cell population, respectively. Changing the inducer concentration not only changed the killing efficiency but also dramatically altered the composition of surviving cell types as well as the distribution of cell death timing. Additionally, multiple rounds of inducer application weakened the killing efficiency due to the accumulation of drug resistance originating from a selective pressure on the low initial iCasp9 level. The killing efficiency on the resistant population could be greatly improved by the combinational use of the inducer with XIAP-targeted drug at the end of the multiple-round inducer treatment. Interestingly,

\footnotetext{
${ }^{1}$ Center for Quantitative Biology and Peking-Tsinghua Center for Life Sciences, Academy for Advanced Interdisciplinary Studies, Peking University, 100871 Beijing, China. ${ }^{2}$ School of Engineering and Applied Sciences, Harvard University, Cambridge, MA 02138, USA. ${ }^{3}$ School of Physics, Peking University, 100871 Beijing, China.

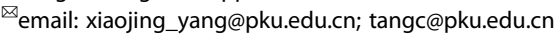

Edited by Professor Boris Zhivotovsky
}

Received: 3 August 2021 Revised: 29 November 2021 Accepted: 10 December 2021

Published online: 10 January 2022 
a

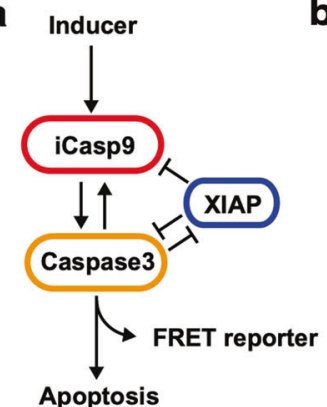

b

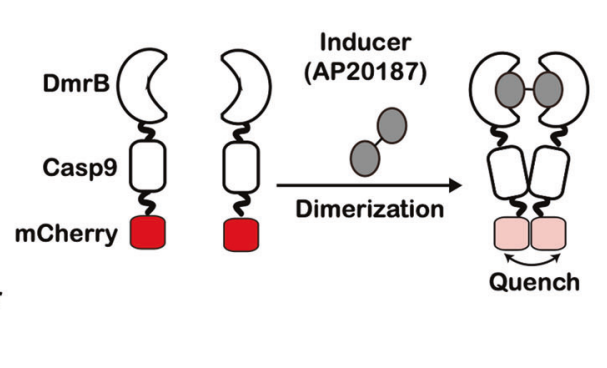

C

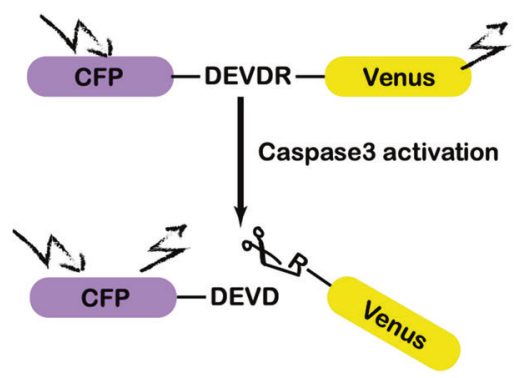

\section{d BF+iCasp9 mCherry merge (input)}

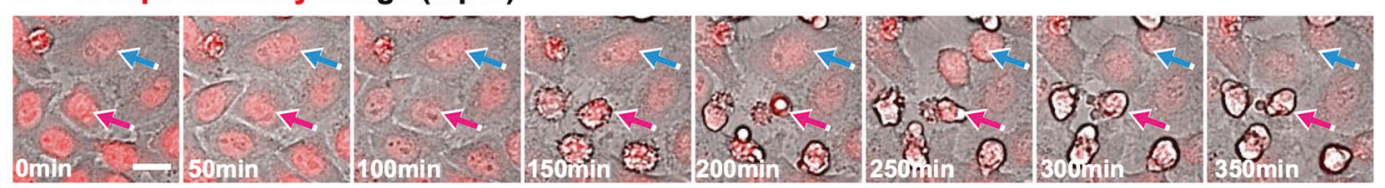

CFP+FRET merge (cell fate reporter)

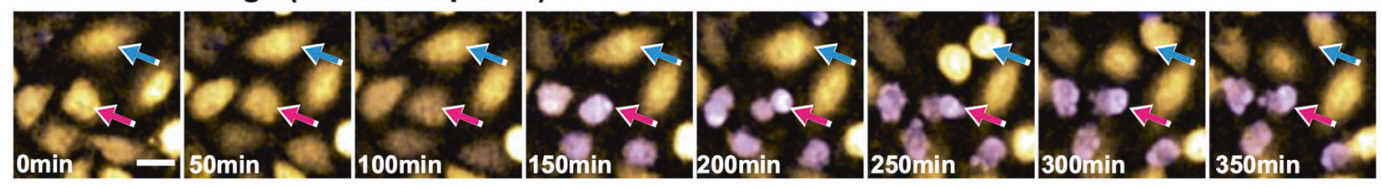

e

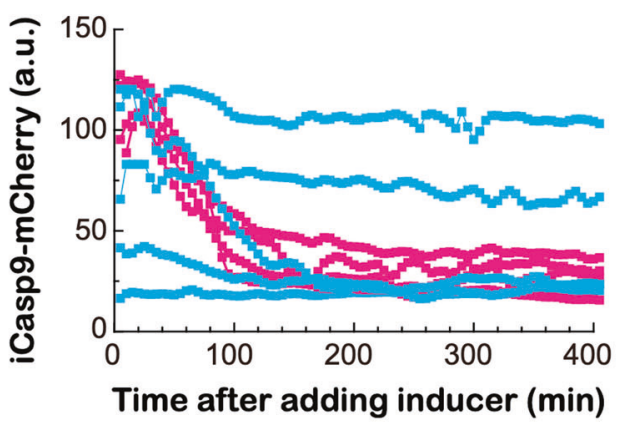

f

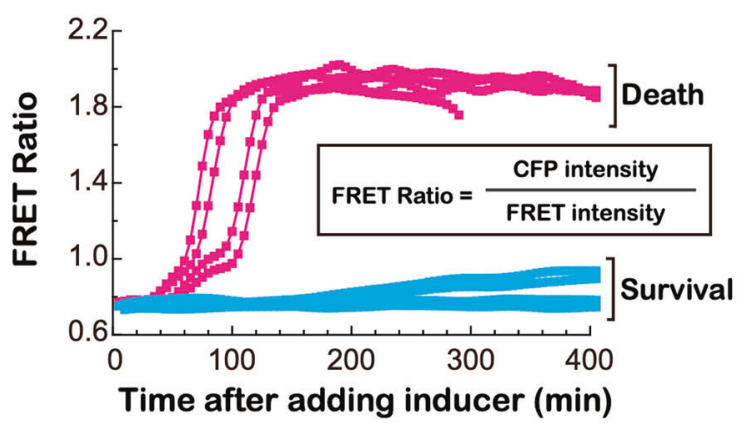

Fig. 1 Construction of iCasp9 system to monitor iCasp9 dimerization and cell fate simultaneously in single cells. a AP20187-induced apoptosis pathway in iCasp9 cells. b Schematic diagram for induction of iCasp9 dimerization. White half-moon shape represents the DmrB domain which can be bound and dimerized by inducer (gray). White square represents full-length Caspase9, which is tagged with an mCherry fluorescence protein (red). Upon dimerization, the iCasp9-mCherry fluorescence signal will be greatly quenched. c The FRET reporter indicating Caspase 3 activity and cell fate. $\mathbf{d}$ Microscopy images of merged BF and iCasp9-mCherry channel (upper panel) and merged CFP and FRET channel (lower panel) for iCasp9 cells treated with $0.25 \mathrm{nM}$ AP20187. The blue and the pink arrow indicates a typical surviving and dead cell, respectively. Images were taken under a $\times 40$ confocal fluorescence microscope. e Single-cell profiles of iCasp9-mCherry fluorescence signals in dead cells (shown in pink) and surviving cells (shown in blue) after addition of $0.25 \mathrm{nM}$ inducer. f Single-cell profiles of FRET Ratio signals in dead cells and surviving cells after addition of $0.25 \mathrm{nM}$ inducer.

the elevation of killing efficiency brought by the combinatorial drug use was much less effective if the drug combination was used from the beginning of the multiple-round treatment. Taken together, our results unveiled the source of cell heterogeneity in iCasp9-mediated cell death and demonstrated the "smart application" of combinational drugging for optimizing the killing efficiency, which may offer insights and new strategies for better clinical use of the iCasp9 system.

\section{RESULTS}

\section{A system to monitor iCasp9 dimerization and cell fate} simultaneously in single cells

To monitor iCasp9 dimerization, we constructed an isogenic iCasp9 Hela cell line, in which iCasp9 was tagged with an mCherry fluorescence protein. As fluorescence signals can be greatly quenched upon dimerization due to the reduction of electronic energy level [27-29], the dimerization of iCasp9 thus could be reflected by the decrease of mCherry fluorescence intensity (Fig. $1 \mathrm{~b}$ and Supplementary Fig. 1a-c).

For the cell fate, since cells will all undergo apoptosis once Caspase 3 is activated, we introduced a Fluorescence Resonance Energy Transfer (FRET)-based reporter, CFP-DEVDR-Venus, to monitor the Caspase3 activation (Fig. 1c) [30]. In this reporter, CFP and Venus are linked together by five amino acids 'DEVDR', which is the optimal substrate sequence for Caspase3. The ratio of CFP versus Venus fluorescence (FRET Ratio) will change upon cleavage of the linker 'DEVDR' (Fig. 1c and Supplementary Fig. 1d-f). The FRET ratio of the reporter is highly correlated with the morphology change of apoptotic cells as we monitored cells under the microscope (Fig. 1d).

Using the iCasp9 cell system above, we characterized heterogeneous cell responses to the inducer. We extracted single-cell profiles of iCasp9-mCherry (Fig. 1e and Supplementary Video 1) and FRET Ratio (Fig. 1 f and Supplementary Video 2) from surviving and dead cells, respectively. A significant cell-to-cell variability was 
observed. Some cells were dead and characterized with a significant decrease of iCasp9-mCherry (Supplementary Video 3) and a sharp increase of FRET Ratio (pink lines, Fig. 1e, $f$ and Supplementary Video 4), while some cells survived with various iCasp9-mCherry profiles but a constantly low FRET Ratio (blue lines, Fig. 1e, $\mathrm{f}$ and Supplementary Video 5).

\section{Heterogenous cell fates dictated by initial iCasp9 level and XIAP/C3 ratio}

To investigate the source of heterogenous cell fates, we compared the profiles of both iCasp9-mCherry and FRET Ratio in surviving cells (Fig. 2a) to those in dead cells (Fig. 2b). As expected, the surviving cells showed a constantly low FRET Ratio, while the dead cells exhibited a sharp increase in the FRET Ratio. Unexpectedly, the dead cells showed a significantly higher initial iCasp9-mCherry level than that in surviving cells (Fig. 2c). We further binned the cells by the initial iCasp9 level, and found a clear dependency of the death percentage on the initial iCasp9 level (Fig. 2d), suggesting the cell fate is highly correlated with its initial iCasp9 level.

However, the death percentage reached a plateau of $\sim 80 \%$ when the initial iCasp9-mCherry level increased to 100 (Fig. 2d). In addition, the initial iCasp9 level in surviving and dead cells had a big overlap, though the difference between them was significant (Fig. 2c). All these results suggested that the initial iCasp9 level is not the sole determinant of the cell fate, which led us to consider the potential effect of the other two players in the AP20187induced apoptosis pathway: XIAP and Caspase3. Thus, we measured their amount in both surviving and dead cells treated with $0.25 \mathrm{nM}$ inducer for different times (Fig. 2e). Compared with the initial values $(0 \mathrm{~h})$, both XIAP and Caspase 3 dramatically decreased after adding inducer for $6 \mathrm{~h}$, even in the surviving cells (Fig. 2f, g), indicating a fighting process between XIAP and Caspase 3 [31]. However, the XIAP/C3 ratios in surviving cells were significantly higher than that in dead cells at all timepoints (Fig. 2h), suggesting that the cell survives due to the overwhelming XIAP to Caspase3 level.

To further confirm the effect of XIAP/C3 ratio on cell fate, we knocked down the level of either XIAP or Caspase3 by RNAi. With a knockdown efficiency of $68 \%$ on XIAP, the death percentage was increased from $68 \%$ to $84 \%$, while a knockdown efficiency of $59 \%$ on Caspase3 led to a decrease of death percentage from 68 to $53 \%$ (Fig. 2i). These results indicate that the variability of XIAP and Caspase3 levels in single cells also contributes to cell fate heterogeneity. Given the numerous XIAP-targeted drugs, these results opened up a potential therapeutic strategy of combining iCasp9 inducer with XIAP inhibitors to achieve a higher killing efficiency. We tested this strategy in our system, using AT406, a small molecule drug, for XIAP inhibition. Indeed, a significant increase in killing efficiency was observed in all inducer concentrations (Fig. 2j).

Taken together, by employing the iCasp9 cell system we constructed, we found that the cell-to-cell variability in the initial iCasp9 level and the XIAP/C3 ratio are the main two contributors for the cell fate heterogeneity.

\section{Different types of surviving cells}

In addition to the heterogenous surviving and dead cell fates, substantial heterogeneity was also found within both the surviving and dead cell populations. As for the surviving cells, we observed several distinct types in light of their iCasp9-mCherry and FRET Ratio profiles, indicating that they survive because of different reasons.

Based on whether there was a decrease in iCasp9-mCherry signal after adding the inducer, surviving cells are divided into two groups. Those with no decrease of iCasp9-mCherry $(<50 \%)$ were grouped as surviving cell type 1 , indicating an insufficient induction of iCasp9 (red lines, Fig. 3a). All type 1 cells showed constantly low FRET Ratio signals (purple lines, Fig. 3a). The insufficient iCasp9 induction was presumably due to either low initial iCasp9 level or low inducer uptake efficiency. Thus, we further classified type 1 surviving cells into two subpopulations according to their initial iCasp9-mCherry levels. Since the dead probability of cells with the initial iCasp9-mCherry level above 60 started to be higher than the survival probability (Supplementary Fig. 2), we grouped cells with initial iCasp9-mCherry level below 60 as surviving cell type1-a (left panel, Fig. 3a) while the remaining cells as surviving cell type $1-b$ (right panel, Fig. 3a).

For those surviving cells with a significant decrease of iCasp9mCherry, we grouped them into surviving cell type 2 (Fig. 3b). In contrast to the constantly low FRET Ratio in surviving cell type 1, we observed a slight increase of FRET Ratio happening concomitantly with the decrease of iCasp9-mCherry in surviving cell type 2, suggesting that Caspase 3 had been partially activated. The final surviving cell fate was reached in these cells presumably due to a high XIAP level overwhelming the apoptotic function of iCasp9 and Caspase3. Based on whether the initial iCasp9 level was higher than 60 , we also classified type 2 cells into two subpopulations, surviving cell type2-a (initial iCasp $9<60$, left panel, Fig. 3b) and surviving cell type2-b (initial iCasp9 $\geq 60$, right panel, Fig. 3b), respectively.

To investigate the contributions of each survival mode, we further quantified the number of surviving cells from different types (Supplementary Fig. 3). Among 44 surviving cells we analyzed under the condition of $0.25 \mathrm{nM}$ inducer, only $22.7 \%$ (10/44) of cells had no obvious iCasp9 dimerization (Fig. 3c, Type 1), and $90 \%(9 / 10)$ of them showed the initial iCasp9 level lower than 60 (Fig. 3d, Type1-a), indicating the importance of initial iCasp9 level for an effective iCasp9 dimerization. The remaining cells $(1 / 10)$ showed reasonably high initial iCasp9 level but no sign of dimerization, suggesting a low efficiency of drug uptake (Fig. 3d, Type1-b) [32]. However, 77.3\% (34/44) of cells presented significant iCasp9 drops (Fig. 3c, Type 2), implying that most of the surviving cells had an effective iCasp9 dimerization. Among this population, $44.1 \%$ (Fig. 3e, Type2-a) and $55.9 \%$ (Fig. 3e, Type2-b) of the cells had initial iCasp9 levels lower and higher than 60, respectively. Although all Type 2 cells survived from the fighting between XIAP and Caspases, fewer cells in Type2-a had an increase of FRET Ratio (>15\%) than that in Type2-b (Fig. 3f), indicating higher initial iCasp9 level would generally lead to higher Caspase3 activation.

\section{Substantial heterogeneity exists within the dead cell population}

Then we looked into the dead cell population. Although a significant drop of iCasp9-mCherry fluorescence and a sharp increase of FRET ratio were observed in all dead cells (Fig. 4a), the dynamics of the iCasp9 dimerization (Fig. 4b) and Caspase3 activation (Fig. 4c) showed a large cell-to-cell variability.

To quantify the heterogeneity, we defined "Td-iCasp9" as the time for iCasp9-mCherry intensity decreasing from the initial level to the level at $10 \%$ higher than the minimum (Fig. 4b), and "Td-dead" as the time from adding inducer to the FRET Ratio reaching $90 \%$ of its maximum (Fig. $4 \mathrm{C}$ and Supplementary Fig. 4), respectively.

As iCasp9 dimerization was the effective input of the circuit, we asked what features of iCasp9 determine the variability of death timing. We first looked at the initial iCasp9 level, which is correlated with cell survival/death fates. However, only a weak negative correlation (Pearson's $r=-0.13$ ) was found between $\mathrm{Td}$ dead and the initial iCasp9 level (Fig. 4d). Then we analyzed the correlation between Td-dead and Td-iCasp9 (Fig. 4e). A strong positive correlation (Pearson's $r=0.74$ ) was seen, indicating cells with faster iCasp9 dimerization are more likely to die faster than cells with slower iCasp9 dimerization. Different dynamics of iCasp9 
a

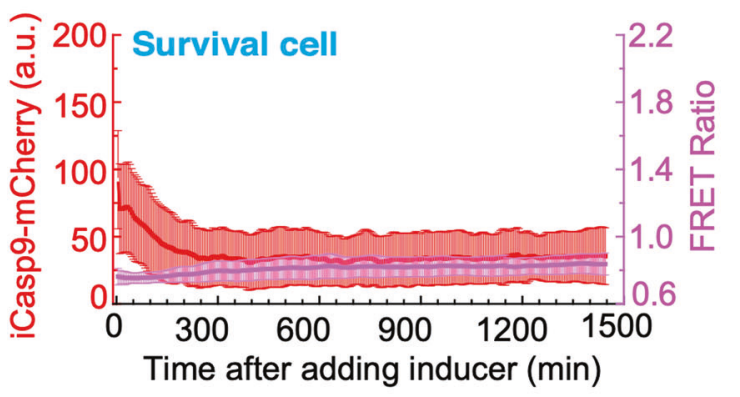

C

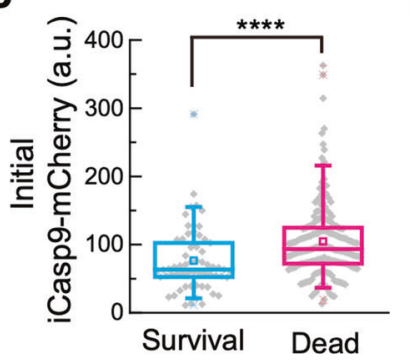

d

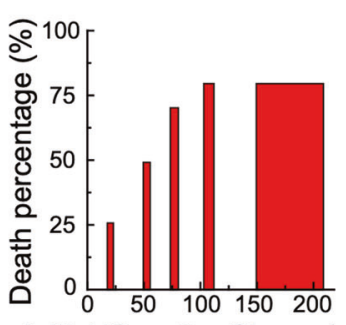

b

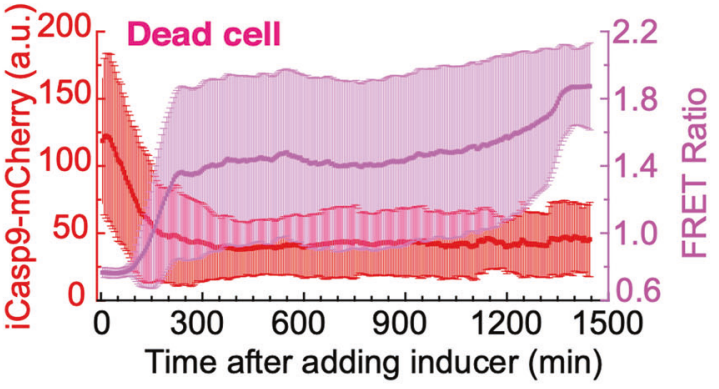

e

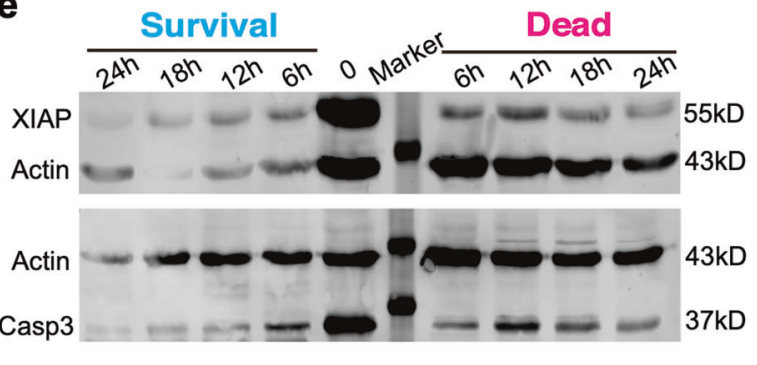

f

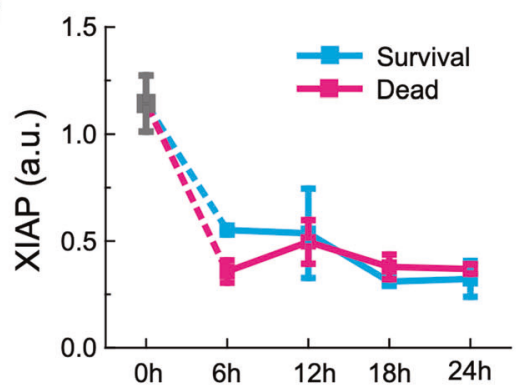

Time after adding inducer g

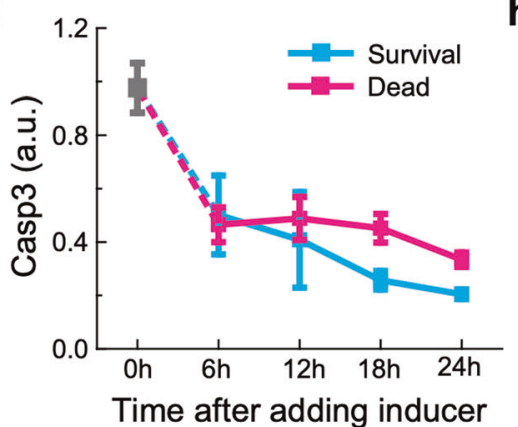

h

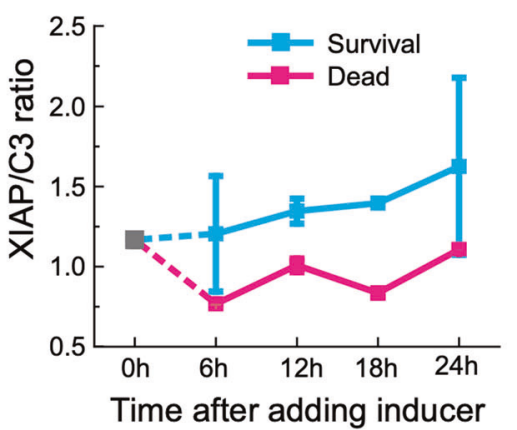

i

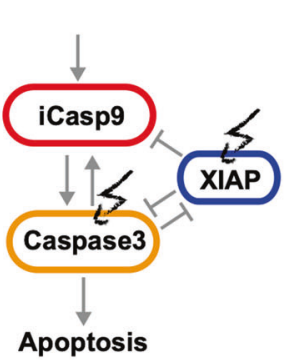

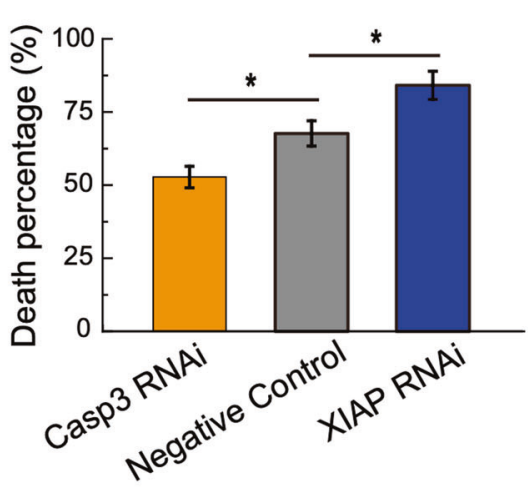

j

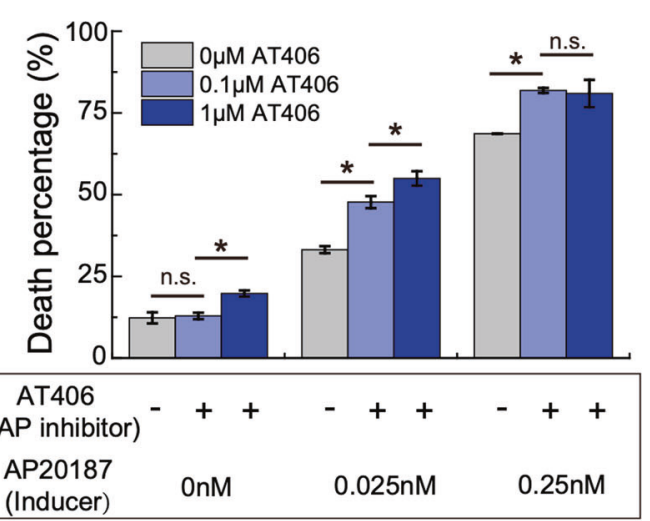

Fig. 2 Initial iCasp9 level and XIAP/C3 ratio in surviving and dead cells. a, b The averaged iCasp9-mCherry fluorescence signal (shown in red) and FRET Ratio signal (shown in purple) in surviving cells (a) and dead cells (b) after treatment of $0.25 \mathrm{nM}$ inducer. The data for surviving cells are averaged from 40 independent measurements, and the data for dead cells are averaged from 68 independent measurements. Error bars represent \pm standard deviation. $\mathbf{c}$ The initial iCasp9-mCherry level in surviving and dead cell populations treated with $0.25 \mathrm{nM}$ inducer. The boxes represent the interquartile range between the first and third quartiles, whereas the whiskers represent the $95 \%$ and $5 \%$ values, and the squares represent the average. $\mathbf{d}$ Cell death percentage is plotted against the initial iCasp9-mCherry expression level. e Western Blot for XIAP (upper panel) and Caspase3 (lower panel) in surviving and dead cells after treating with $0.25 \mathrm{nM}$ inducer for $6,12,18$, and $24 \mathrm{~h}$. Actin was used as a loading control. f-h Quantitative analysis of XIAP level (f), Caspase3 level (g), and XIAP/C3 ratio (h) in surviving cells (blue) and dead cells (pink) in different timepoints. i Perturbations of XIAP or Caspase3 expression level by siRNA. Death percentage for cells treated with XIAP siRNA (blue) or Caspase3 siRNA (orange) is compared with a negative control (gray). $\mathbf{j}$ Death percentage for cells treated with different concentrations of an XIAP inhibitor AT406 in combination with $0.025 \mathrm{nM}$ inducer (light blue), $0.25 \mathrm{nM}$ inducer (blue) and no inducer (gray). Error bars represent \pm standard deviation. ${ }^{*} P<0.05 ;{ }^{* * *} P<0.0001$. 
a

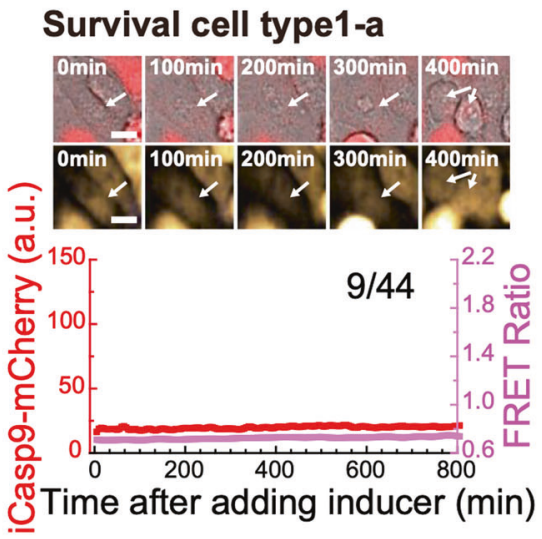

b

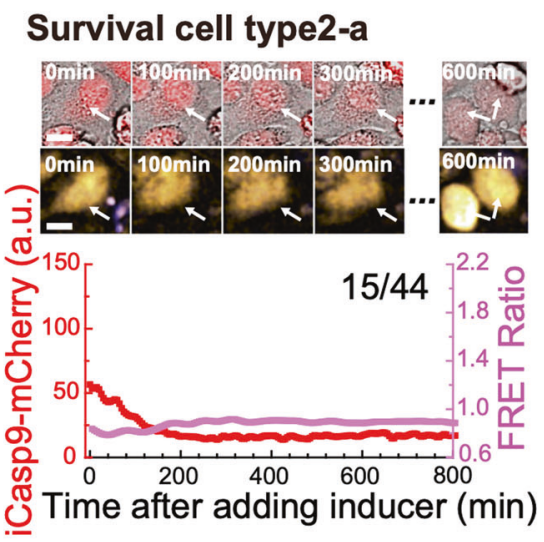

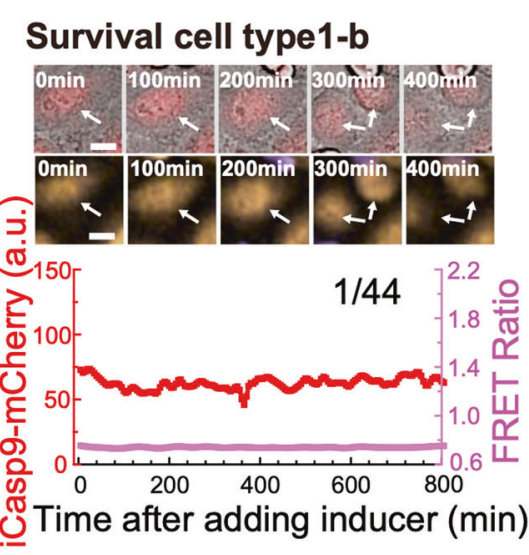

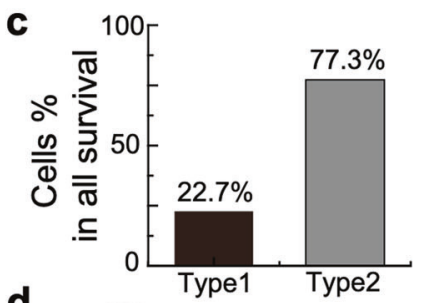

d

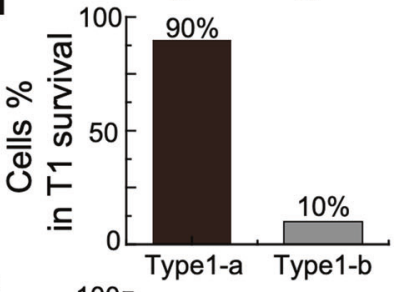

\section{Survival cell type2-b}

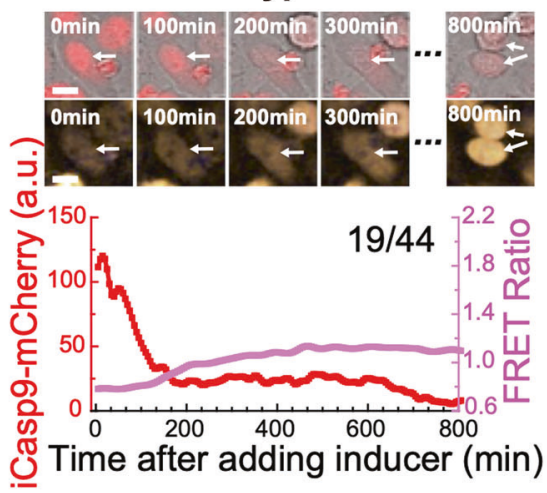

e

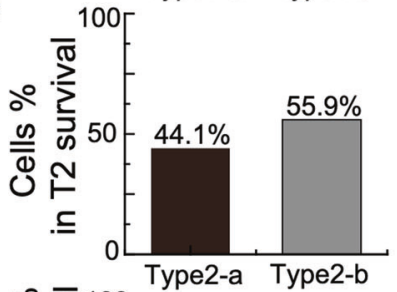

$f$

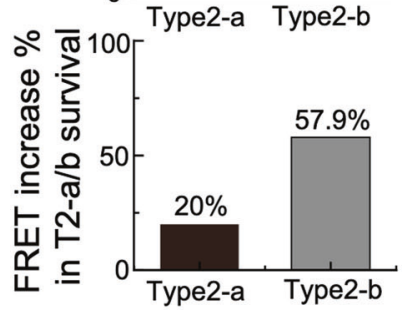

Fig. 3 Substantial heterogeneity exists within surviving cell population. $\mathbf{a}, \mathbf{b}$ Different types of cells surviving from the treatment of inducer. The upper panels show representative images (BF and iCasp9-mCherry merge on top, CFP and FRET merge on bottom) of different surviving cell types. The lower panels show the trajectory of iCasp9 dimerization (red) and Caspase 3 activation (purple) measured from the fluorescence images above. (a, left) A surviving cell with low initial iCasp9-mCherry level and no iCasp9-mCherry drop, and a constantly low FRET Ratio. (a, right) A surviving cell with high initial iCasp9-mCherry level and no iCasp9-mCherry drop, and a constantly low FRET Ratio. (b, left) A surviving cell with low initial iCasp9-mCherry level and an iCasp9-mCherry drop, and a constantly low FRET Ratio. (b, right) A surviving cell with high initial iCasp9-mcherry level and an iCasp9-mCherry drop, and a slight increase of FRET Ratio. c The percentage of type 1 and type 2 surviving cells in all surviving cells studied. $\mathbf{d}$ The percentage of type1-a and type1-b surviving cells in type1 surviving cells. e The percentage of type2-a and type2-b surviving cells in type 2 surviving cells. $f$ The percentage of surviving cells with FRET Ratio increase by at least $15 \%$ of the initial ratio in type2-a and type2-b surviving cells.

dimerization were presumably due to the cell-to-cell variability on drug transportation efficiency [32].

\section{Dose-effect of inducer on caspase dynamics and cell fates}

Since drug dose is the easiest thing to be adjusted in the clinic, we further varied the inducer concentration and investigated the dose-effect on cellular responses. We first systematically investigated the killing efficiency of different inducer concentrations, ranging from 0.0001 to $100 \mathrm{nM}$ (Fig. 5a and Supplementary Fig. 5). While the wild-type cells which do not incorporate iCasp9 gene did not respond to the inducer (black dots, Fig. 5a), iCasp9 cells showed a significant enhancement of cell death with increasing inducer concentration (red dots, Fig. 5a). To understand the doseeffect of inducer at single-cell level, we further looked into the dynamics of iCasp9 dimerization and Caspase 3 activation.

We first extracted the average trajectories of iCasp9-mCherry from all dead (Fig. 5b) and surviving cells (Fig. 5c). Obvious differences were found both between dead and surviving cells and among different concentrations. The surviving cells showed a significantly lower initial iCasp9 level than that in dead cells under concentrations of $0.25 \mathrm{nM}$ and $2.5 \mathrm{nM}$, further confirming our conclusion that cells with higher initial iCasp9 levels are more easily die (Fig. 5 d). No significant difference on initial iCasp9 level was found between dead and surviving cells in the concentration of $0.025 \mathrm{nM}$, presumably due to the extremely low killing efficiency at that concentration, which was just slightly higher than the background death percentage (Fig. 5a). In this case, the contribution to cell fate from other factors, such as the XIAP and Caspase 3 level, may shadow that of the initial iCasp9 level. It is worth noting that a decreasing initial iCasp9 level was found in surviving cells as the inducer concentration increases, indicating that some surviving cells with high initial iCasp9 levels can be killed by increasing the inducer concentration (Blue dots, Fig. 5d).

As for the dynamics of iCasp9 dimerization, we measured the Td-iCasp9 for surviving and dead cells under different inducer concentrations. For surviving cells, since there was no obvious iCasp9 dimerization in type 1 cells, we only extracted the averaged trajectories of iCasp9-mCherry from type 2 cells for Td-iCasp9 analysis (Supplementary Fig. 6a). It turned out that, even type 2 cells had iCasp9 dimerization, their Td-iCasp9 was significantly longer than that in dead cells, suggesting that surviving cells may generally have a weak capability in drug uptake. Moreover, both surviving and dead cells possessed a shortened Td-iCasp9 when being treated with higher concentrations, suggesting higher inducer concentration would speed up the iCasp9 dimerization.

To further support our findings that faster iCasp9 dimerization can accelerate cell death, we extracted the trajectories of FRET Ratio in each dead cell under different inducer concentrations 


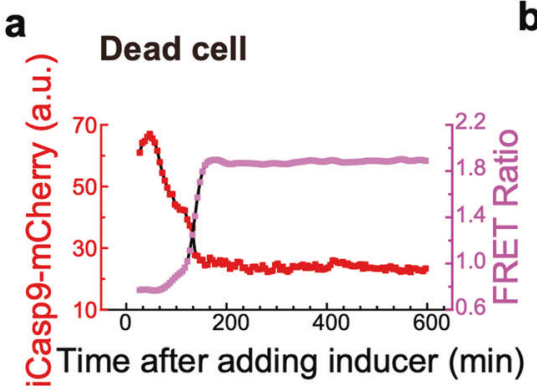

b

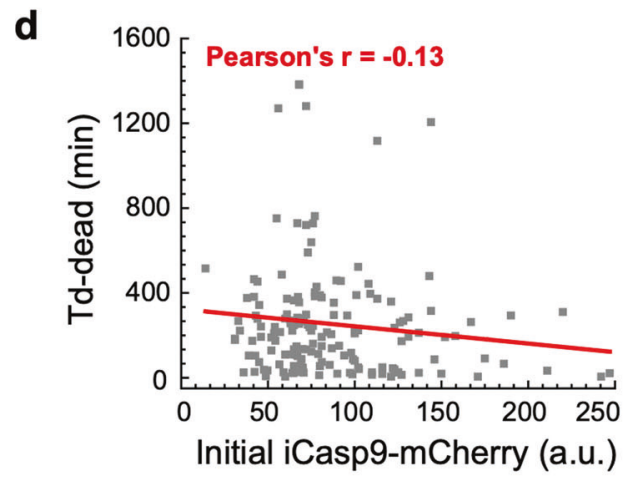

Dead cell - iCasp9 mCherry

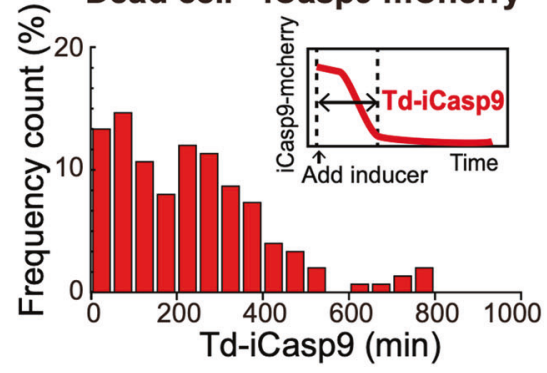

c

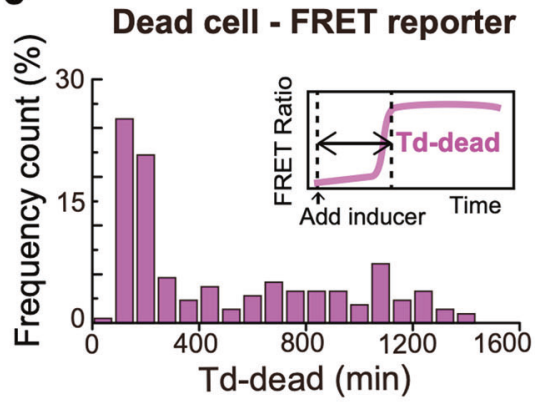

e

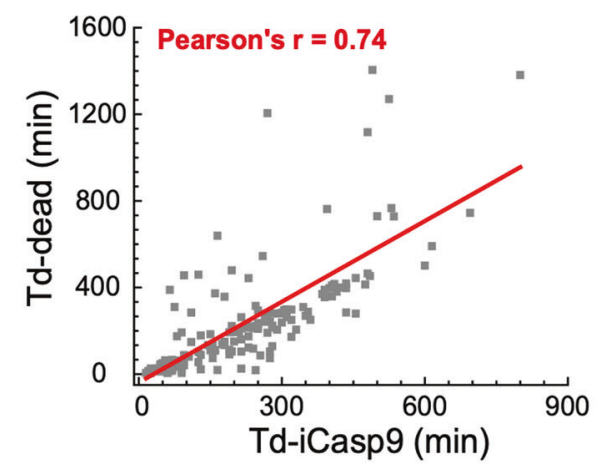

Fig. 4 Cell-to-cell variability of death timing in dead cell population. a Fluorescence signals of iCasp9-mCherry and FRET ratio in a typical dead cell. b Frequency count of dead cells with different iCasp9 dimerization time under the condition of $0.25 \mathrm{nM}$ inducer. Inner image represents a schematic view for iCasp9 dimerization time 'Td-iCasp9', which is defined as the time duration from adding inducer to the iCasp9mCherry fluorescence signal reaching $10 \%$ higher than the minimum. c Frequency count of dead cells with different death time under the condition of $0.25 \mathrm{nM}$ inducer. Inner image represents a schematic view for cell death time 'Td-dead', which is defined as the time duration from adding inducer to the FRET ratio reaching $90 \%$ of the maximum. $\mathbf{d}$ Td-dead is plotted against the initial iCasp9-mCherry level in dead cells. Pearson's correlation is -0.13 . e Td-dead is plotted against Td-iCasp9 in dead cells. Pearson's correlation is 0.74 .

(left panel, Fig. 5f). Quantitative analysis on Td-dead (right panel, Fig. 5f) showed that cells died at an averaged time of $\sim 800 \mathrm{~min}$ after being treated with $0.025 \mathrm{nM}$ inducer, with the 5th and 95th percentile values of $300 \mathrm{~min}$ and $1400 \mathrm{~min}$. However, in the condition of $2.5 \mathrm{nM}$ inducer, the averaged Td-dead was dramatically shortened to $\sim 80 \mathrm{~min}$ and the variability was also greatly reduced. These results led us to propose that slow dimerization of iCasp9 resulted in a gradual accumulation of active iCasp9, which might be easily neutralized by XIAP and thus hampers cell death. We further quantified Td-iCasp9 and cell death percentage in different inducer concentrations. The results showed cell death percentage was negatively related to Td-iCasp9, confirming that rapid iCasp9 dimerization is more potent to causing cell death (Supplementary Fig. 6b).

Besides the impact on death timing in dead cells, drug dose also changed the composition of surviving cell types. We found the surviving cells with low initial iCasp9 levels became dominant in higher concentrations (type1-a + type2-a, Fig. 5g).

Taken together, the higher concentration of AP20187 would speed up the iCasp9 dimerization, and lead to faster death with lower cell-to-cell variability.

\section{Development of drug resistance in multiple rounds of inducer treatment}

We exposed iCasp9 cells to multi-rounds of $0.25 \mathrm{nM}$ inducer treatment (Fig. 6a). Instead of approaching higher killing efficiency, an obvious accumulation of drug resistance was observed with repeated rounds of treatment. While $65 \%$ of cells died after the first round of treatment, the death percentage dropped to $48 \%$ for the second round and only $12.3 \%$ of cells were killed in the 3 rd round treatment (Fig. 6b).

To further investigate the origin of the accumulating drug resistance, we quantified the iCasp9, XIAP, and Caspase3 protein levels in cells right before each round of inducer treatment. A significant drop of the initial iCasp9-mCherry level was observed as the round increases, indicating a strong selective pressure on the initial iCasp9-mCherry level (Fig. 6c and Supplementary Fig. 2b). Meanwhile, we also found a decrease in Caspase3 level, while XIAP maintained at a similar level (Fig. 6d-f). As a result, the XIAP/ C3 ratio was higher in cells that survived multiple rounds of treatment (Fig. 6g).

As discussed before, cells survive from the inducer treatment in different ways (Fig. 3). We compared the composition of different surviving cell types after the first and the third round of treatment (Fig. 6h). While there was a total of 50\% (type1-a + type2-a) surviving cells (initial iCasp9 $<60$ ) after the first treatment (left pie chart, Fig. $6 \mathrm{~h}$ ), the percentage increased to $77 \%$ after three rounds of treatment (right pie chart, Fig. 6h). These results further support the selective pressure on the initial iCasp9 level. Interestingly, the percentage of surviving cells due to high XIAP/C3 ratio (type2-b) dropped dramatically from 47 to $15 \%$, suggesting minor contributions of XIAP/C3 ratio to the increasing drug resistance, consistent with the fact that no obvious selective pressure was observed on XIAP level (Fig. 6f). In terms of why there was no obvious selection pressure on XIAP being found, it was presumably due to the fact that the probability of mutation to upregulate a protein level (XIAP) is lower than that of downregulating a protein level (or disfunctioning a protein) (iCasp9 and Caspase3). Taken together, our experiments showed that multiple rounds of treatment cannot increase the killing efficiency. Instead, it would place selective pressure on protein levels, especially on the level of initial iCasp9, leading to drug resistance.

Since no obvious change on XIAP level was found after multiple rounds of treatment, we wonder if higher killing efficiency could be achieved by combining inducer with XIAP inhibitor. Indeed, after two rounds of AP20187 treatment, by combining with $0.1 \mu \mathrm{M}$ 


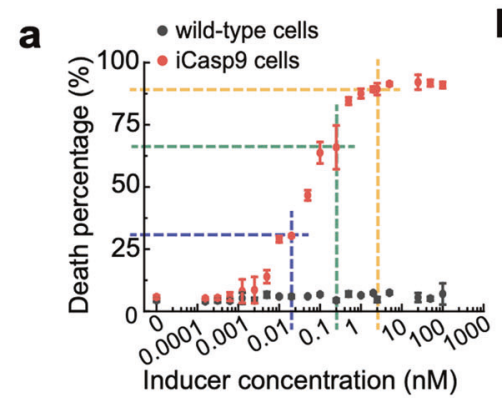

d

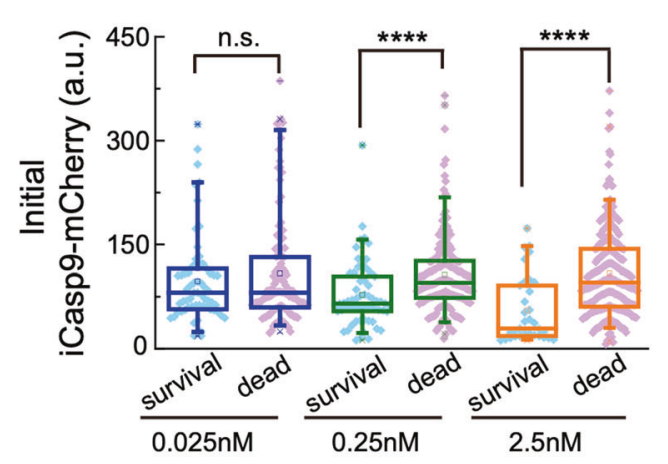

C

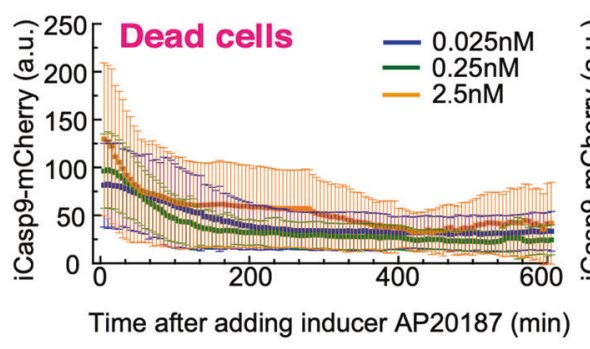

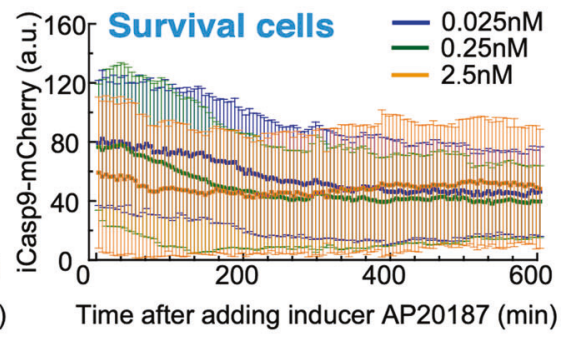

Q

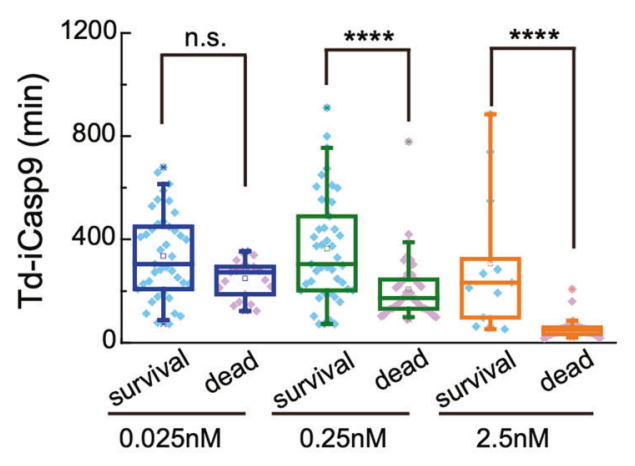

$\mathbf{f}$
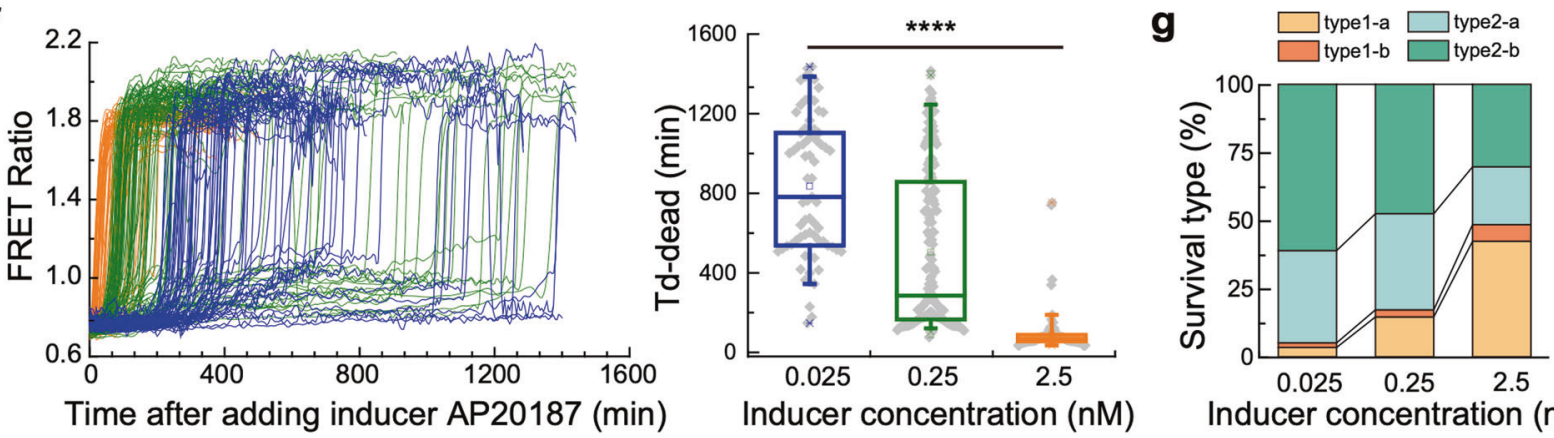

Inducer concentration (nM)

Fig. 5 Inducer dosage affects caspase dynamics, cell-to-cell variability, and cell death. a Cell death percentage is plotted against different concentrations of inducer. Data are shown as mean \pm standard deviation. b, c Averaged trajectories of iCasp9-mCherry for dead cells (b) and surviving cells (c) treated with $2.5 \mathrm{nM}$ (orange), $0.25 \mathrm{nM}$ (green) and $0.025 \mathrm{nM}$ (dark blue) inducer. Error bars represent \pm standard deviation. d, e Initial iCasp9-mCherry level (d) and Td-iCasp9 (e) in surviving (blue dots) and dead (pink dots) cells after the treatment of 0.025, 0.25, and $2.5 \mathrm{nM}$ inducer. $\mathbf{f}$ The trajectories of FRET Ratio for individual dead cells in the condition of $0.025,0.25$, and $2.5 \mathrm{nM}$ inducer (f, left panel). Tddead for dead cells treated with $0.025,0.25$, and $2.5 \mathrm{nM}$ inducer (f, right panel). $\mathbf{g}$ Percentage of four survival cases in cell population treated with $0.025,0.25$, and $2.5 \mathrm{nM}$ inducer. The boxes of the boxplot represent the interquartile range between the first and third quartiles, whereas the whiskers represent the $95 \%$ and $5 \%$ values, and the squares represent the average. ${ }^{*} P<0.05 ;{ }^{* *} P<0.01,{ }^{* * *} P<0.001,{ }^{* * * *} P<0.0001$.

XIAP inhibitor AT406 at the third round, a significant increase of killing efficiency was obtained, from 16.5 to $62.4 \%$ (gray bars, Fig. 6i). Surprisingly, if the drug combination was used from the beginning, i.e., if the previous two rounds of treatment were by the combination of inducer AP20187 and XIAP inhibitor AT406, the killing efficiency at the third round was much lower with or without the drug combination (yellow-green bars, Fig. 6i). This suggests that the continuous application of combinational drugging placed selective pressure on both iCasp9 and XIAP, resulting in a dramatic attenuation of the efficacy of the XIAP inhibitor. Our findings hint at a "smart strategy" of drug combination usage.

\section{DISCUSSION}

Our analysis indicated that the killing efficiency could be adjusted by changing the protein expression level. Indeed, the killing efficiency was increased with a knockdown on XIAP, while a knockdown on Caspase3 led to a decrease in the death percentage. Thus, using a vector to achieve higher iCasp9 expression would presumably overcome some of the drug resistance. These results suggest a big difference in the killing efficiency for various cell types or tissues/organs, as their protein expressions including Caspase3, Caspase9, and XIAP varies a lot (seen in database 'The Human Protein Atlas'). Therefore, personalized treatment with respect to specific cell types or tissues may be needed to reach the desired killing efficiency.

Multiple rounds of inducer application did not kill more cells. Instead, the surviving cells developed heritable drug resistance. We found that the resistance came from the low iCasp9 expression. This heritable protein variation probably originated from the epigenetic differences [33] in individual cells. Further investigation of the epigenetic source may improve iCasp9mediated killing efficiency. Interestingly, little selective pressure on XIAP expression level was observed in the multi-round inducer treatment. Thus, we can significantly improve killing efficiency on the inducer resistant cell population by combining XIAP-targeted inhibitor with the inducer. It is worth noting that the efficacy of drug combinations was dramatically diminished if used continuously from the very beginning. 


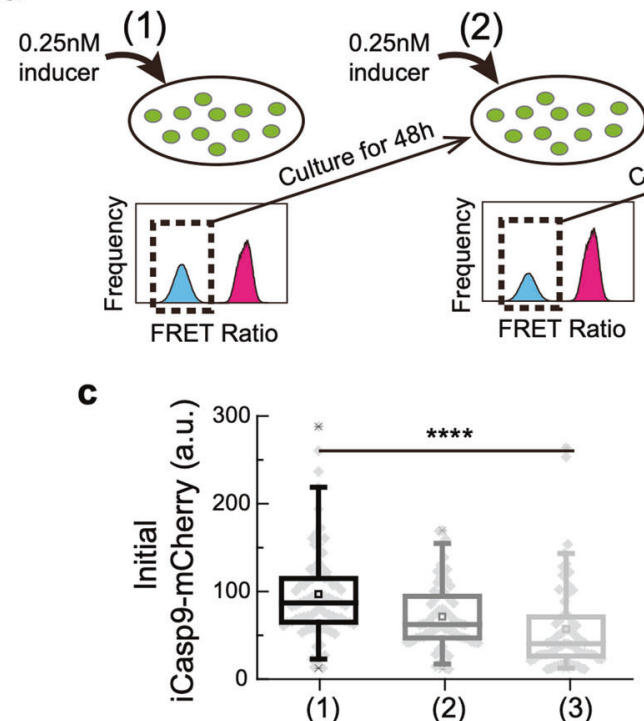

(3)

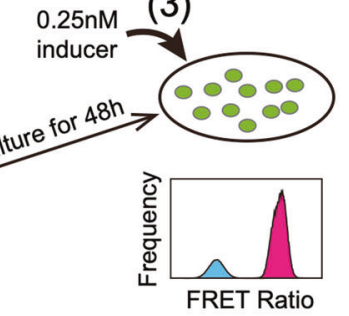

b

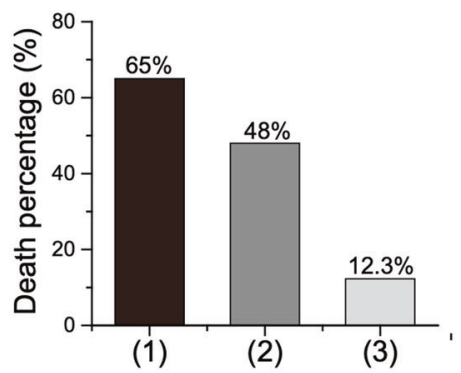

d

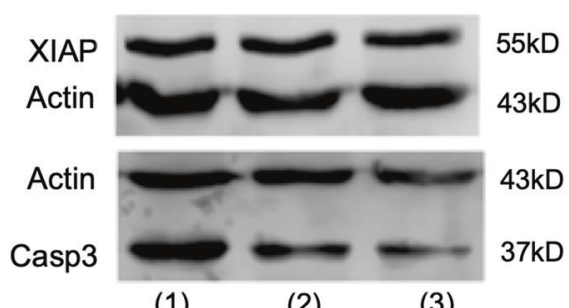

(1) (2) (3)

f

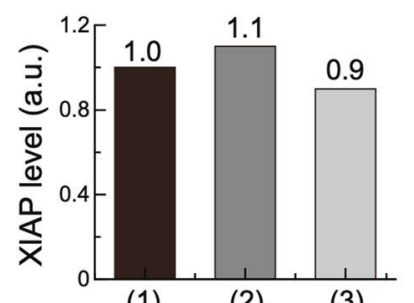

g

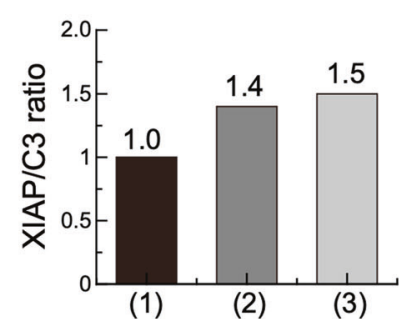

h

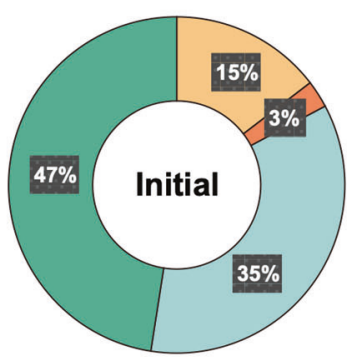

$\square$ Survival type1-a

Survival type1-b

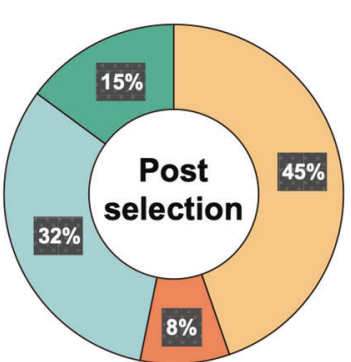

Survival cell type2-a

Survival cell type2-b i

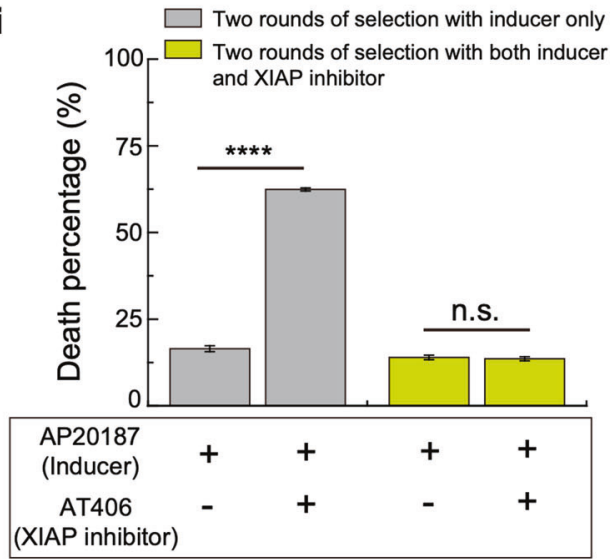

Fig. 6 Multiple rounds of inducer treatment place selective pressure on protein level and lead to drug resistance. a Schematic view of the multi-drugging experiment. Surviving cell population with low FRET Ratio (shown in blue) is collected and re-cultured for 2 days before the next round of drugging. b Death percentage for cells after each round of treatment with $0.25 \mathrm{nM}$ inducer. c Initial iCasp9-mCherry expression in cells before each round of inducer treatment. The boxes represent the interquartile range between the first and third quartiles, whereas the whiskers represent the $95 \%$ and $5 \%$ values, and the squares represent the average. $\mathbf{d}$ Western Blot for XIAP (upper panel) and Caspase 3 (lower panel) in cells before the first (column 1), the second (column 2), and the third (column 3) round of inducer treatment. Actin was used as a loading control. e-g Quantitative analysis of Caspase3 expression (e), XIAP expression (f), and XIAP/C3 ratio (g) in cells before each round of inducer treatment. $\mathbf{h}$ Percentage of four surviving cell types after the first treatment (left pie chart) and after the third treatment (right pie chart) of the inducer. i Death percentage of post-selection cells at the third round of treatment with either the inducer only or the combination of the inducer and the XIAP inhibitor. The cells were selected by two rounds of treatment with $0.25 \mathrm{nM}$ inducer only (gray bars) or with a combination of $0.25 \mathrm{nM}$ inducer and $0.1 \mu \mathrm{M}$ XIAP inhibitor AT406 (yellow-green bars). Data are shown as mean \pm standard deviation.

The clinic application of iCasp9 suicide gene is still in its infancy. More investigations on the system are required to unleash its full potential. Our work unveils the sources of cell-to-cell variability and drug resistance in the clonal population of iCasp9 cells, paving the way for improving therapeutic strategies to achieve optimal performance.

\section{MATERIALS AND METHODS}

\section{Cell lines and cell culture}

The human embryonic kidney 293 T cells and Hela cervical cancer cell lines were gifts from Prof. Jianguo Chen. Cells were cultured in Dulbecco's Modified Eagle's Medium (DMEM) (Gibco, Cat\# 11965092) containing 10\% fetal bovine serum (Gibco, Cat\# 16000044) and $100 \mathrm{U} / \mathrm{mL}$ penicillin and 
streptomycin (Invitrogen, Cat\# 15140163) in a humidified environment with $5 \% \mathrm{CO}_{2}$ at $37^{\circ} \mathrm{C}$.

\section{DNA constructs}

Lentivirus vectors pHR-mCherry, pCMVdR8.91, and pMD2.G were kindly provided by Prof. Ping Wei. Full-length Caspase9 was a gift from Prof. Xiaodong Wang. The FRET reporter (pECFP-DEVDR-Venus, Cat \# 24537) was purchased from Addgene and the iDimerize ${ }^{T M}$ Inducible Homodimer System (Cat \# 635068) was purchased from Clontech Laboratories, Inc. iCasp9 construct was built by inserting full-length Caspase9 to pHom-1 vector with a linker sequence 'RPPPR', which was verified by sequencing with primers Seq_Casp9_F and Seq_Casp9_R. Then the integrated DmrBlinker-caspase9 sequence in the $\mathrm{pHom}-1$ vector was cloned into the $\mathrm{pHR}$ mCherry vector. We used Primer Spel-Casp9_F and BamHI-Casp9_R to generate a full-length Caspase9 fragment with Spel and BamHI restriction enzyme cleavage site so that we can insert it into the pHom-1 vector, which has the same cleavage site. Primers for constructing and verifying DmrB-linker-caspase9 are listed below.

\begin{tabular}{|c|c|}
\hline Primer name & Primer sequence $\left(5^{\prime}\right.$ to $\left.3^{\prime}\right)$ \\
\hline Spel-Casp9_F & $\begin{array}{l}\text { GGACTAGT }^{\mathrm{a}} \text { AGGCCGCCGCCGAGG } \\
\text { CGAAGCGGATCGGCG }\end{array}$ \\
\hline BamHI-Casp9_R & CGCGGATCC $^{\mathrm{a}}$ CGCTGATGTITAAAGAAAA \\
\hline Seq_Casp9_F & ATGGCTTCTAGAGGAGTGCA \\
\hline Seq_Casp9_R & CGCGGATCCCGCTGATGTITIAAAGAAAA \\
\hline
\end{tabular}

${ }^{a}$ Restriction enzyme cleavage sites are shown in black bold.

${ }^{\mathrm{b}}$ The linker sequence is shown in italics.

\section{Stable cell line construction}

We transfected the FRET reporter into cells by using X-tremeGENE HP DNA Transfection Reagent (Roche, Cat\# 6366236001). We mixed $1 \mu \mathrm{g}$ of plasmid DNA (pECFP-DEVDR-Venus) and $3 \mu \mathrm{l} X$-tremeGENE HP DNA Transfection Reagent with $100 \mu \mathrm{l}$ opti-MEM (Gibco, cat\# 31985088), and added into cells. After transfection, we cultured the cells with DMEM medium containing antibiotic G418 (Sigma, 10131027), starting with the concentration of $1000 \mu \mathrm{g} / \mathrm{ml}$ and then decreasing to maintain the concentration at $200 \mu \mathrm{g} / \mathrm{ml}$ for $\sim 30$ days. Finally, we collected surviving cells and used FACS to select and allocate single cells with positive Venus fluorescence signals into 96-well plates, and cultured every single cell into a big population as the clonal population.

Based on the stable Hela cell line with FRET reporter, the iCasp9 gene was further introduced by lentivirus infection, which was pre-generated from $293 \mathrm{~T}$ cells. We first co-transfected three lentivirus vectors pHRCaspase9-mCherry, pCMVdR8.91, and pMD2.G into 293 T cells. After incubation in $5 \% \mathrm{CO}_{2}, 37^{\circ} \mathrm{C}$ incubator overnight, we harvested the supernatant which contains the lentivirus particles and added them to Hela cells. Two days later, we replaced the medium with a fresh one and kept culturing cells for another week in media containing $0.5 \mathrm{mg} / \mathrm{ml}$ puromycin. The clonal population of iCasp9 cells was established as described above.

\section{siRNA transfection}

The sequence of XIAP siRNA and Caspase3 siRNA was designed for targeting XIAP and Caspase3, respectively. NC siRNA was designed as the negative control. $100 \mathrm{nM}$ siRNA was transfected into iCasp9 cells by using ROCHE X-tremeGENE ${ }^{\mathrm{TM}}$ siRNA Transfection Reagent (MilliporeSigma, Cat\# 4476093001). Cells were harvested $48 \mathrm{~h}$ after transfection and analyzed by western blotting.

\begin{tabular}{ll}
\hline siRNA name & Sequence $\left(\mathbf{5}^{\prime}\right.$ to $\left.\mathbf{3}^{\prime}\right)$ \\
XIAP siRNA & CCAUGUGCUACACAGUCAUTT \\
Caspase3 siRNA & AAUGACAUCUCGGUCUGGUAC \\
NC SiRNA & UUCUCCGACGUGUCACGUTT
\end{tabular}

\section{Western blotting}

For analysis of RNAi knockdown efficiency of XIAP and Caspase3, cells were harvested $48 \mathrm{~h}$ after transfection. For analysis of XIAP and Caspase 3 at different timepoints, we collected surviving and dead cells separately at time $6 \mathrm{~h}, 12 \mathrm{~h}, 18 \mathrm{~h}$, and $24 \mathrm{~h}$ after the treatment of AP20187. Cells were lysed in lysis buffer $(100 \mathrm{mM} \mathrm{NaCl}, 50 \mathrm{mM}$ Tris pH 7.5, 0.5\% deoxycholate, $1 \%$ Triton X-100, $0.1 \%$ SDS). Proteins were run on $12 \%$ Bis-Tris gels (cat\# SL1120-500ML), then transferred to a PVDF membrane (cat\# ISEQ00010) and incubated in a blocking solution (10\% Skimmed Milk Powder, cat\# SUP003a) $2 \mathrm{~h}$ at room temperature. Membranes were then incubated with the primary antibody in Can Get Signal Solution1 (cat\# NKB-101) overnight at $4{ }^{\circ} \mathrm{C}$. Blots were washed three times for $5 \mathrm{~min}$ in washing solution $(50 \mathrm{mM}$ Tris-Cl pH 7.5, $150 \mathrm{mM} \mathrm{NaCl}, 0.05 \%$ Tween-20) and then incubated in blocking solution plus fluorescent secondary antibody in Can Get Signal Solution2 for $1 \mathrm{~h}$. Membranes were washed three times in a washing solution, and we detected protein levels with fluorescent signals (LI-CORODYSSEY CLx Infrared Imaging System). Molecular weights were identified using a protein standard (BioLabs, Cat\# P7712S). Beta-actin was used as the loading control. Antibodies used for western blotting are listed below:

- Primary antibody for XIAP (Cell Signaling Technology, Cat\# 2042 S)

- Primary antibody for Caspase3 (cat\# 9662 S)

- Primary antibody for Caspase9 (cat\# 9502 S)

- Primary antibody for Actin (cat\# 3700 S)

- Secondary antibody (anti-mouse cat\# 926-32211 \& anti-rabbit cat\# 926-68070)

\section{Multiple rounds of inducer treatment}

We exposed iCasp9 cells to multi-rounds of $0.25 \mathrm{nM}$ inducer treatment. After the first round of treatment, surviving cells remained attached to the dish whereas dead cells detached, allowing us to recover surviving cells by trypsinization. We plated surviving cells into a fresh medium without an inducer and cultured them for $48 \mathrm{~h}$. Then these surviving cells were challenged with a second dose of $0.25 \mathrm{nM}$ inducer. We cultured cells that survived from the second dose for another $48 \mathrm{~h}$ and treated them with the third dose of $0.25 \mathrm{nM}$ inducer. For each round of inducer treatment, cells were treated with inducer for $24 \mathrm{~h}$ and the death percentage was monitored.

\section{Flow cytometry analysis}

Flow cytometry FACS Aria (Becton-Dickenson, USA) was used to perform sorting for iCasp9 cell lines. We used a $100 \mu \mathrm{m}$ nozzle and set the forward scatter (FSC) and side scatter (SSC) to 300 and $240 \mathrm{~V}$, respectively, to identify the population of single live cells. We set the short-RFP fluorescence parameter to $500 \mathrm{~V}$, to identify the population of iCasp9mCherry cells. We set the BV421 and BV510 fluorescence parameters to 380 and $320 \mathrm{~V}$, respectively, to identify the fluorescence signals of CFP, FRET, and Venus.

We used the high-throughput mode of flow cytometry to determine cell death percentage. The ratio of CFP to FRET was used to identify live from dead ones. Cells were plated in 96-well flat-bottom plates (Corning) with a density of 40,000 per well. Cells were then treated with AP20187 (Clontech Laboratories, Cat \# 635059) in different concentrations after culturing overnight. After incubation for $24 \mathrm{~h}$, cells were first centrifuged at 2000 rpm for $5 \mathrm{~min}$ to avoid any loss of dead cells. Then we trypsinized and collected all the cells with $200 \mu \mathrm{l}$ medium per well of a 96-well plate as samples. FlowJo software was used to analyze death percentage based on the FRET Ratio and light scattering.

\section{Time-lapse microscopy}

All images were captured by a Nikon Ti inverted microscope equipped with $\times 40 / 0.95$ Plan Apo (Numerical aperture 1.4) objective lens and the Perfect Focus System (Nikon Co., Tokyo, Japan) for continuous maintenance of the focus. Cells were plated in 4-chamber glass-bottom dishes (In Vitro Scientific) with a density of 10,000 per well and maintained in a $37^{\circ} \mathrm{C}, 5 \%$ $\mathrm{CO} 2$ incubation chamber for $24 \mathrm{~h}$ imaging. We used filter sets that are optimized for the detection of mCherry, CFP, FRET, and Venus fluorescence. CFP fluorescence was excited at $440 \mathrm{~nm}$ with a $100 \mathrm{~mW}$ solid-state laser and collected with a 485/60 emission filter (Chroma Technology Corp). 
We set the exposure time at $500 \mathrm{~ms}$. FRET signal was excited by $440 \mathrm{~nm}$ laser with an exposure time of $500 \mathrm{~ms}$ and collected with a 550/49 emission filter. Venus fluorescence was excited at the wavelength of $514 \mathrm{~nm}$ with a $100 \mathrm{~mW}$ solid-state laser and collected with a 550/49 emission filter. The mCherry fluorescence was excited at $561 \mathrm{~nm}$ with a $100 \mathrm{~mW}$ solid-state laser and collected with a 615/70 emission filter (Chroma Technology Corp). All images were captured at an interval of $5 \mathrm{~min}$ for $24 \mathrm{~h}$

\section{Image processing}

We used ImageJ (National Institute of Health), Cellprofiler (Broad Institute), and Matlab (Mathworks) for image processing. ImageJ was used to subtract the background of images and transform the image format to adapt the software Cellprofiler. Cell segmentation and tracing were accomplished by the Cellprofiler pipeline. Firstly, cell boundary was identified based on the Venus signal to track cells at every time point. Then, we quantified the mean intensity of CFP, FRET, and Venus signals in each cell for every time point. Finally, all these measurements were exported as EXEL files for the following analysis. Further analysis was performed by the Matlab. We extracted trajectories of FRET Ratio and iCasp9-mCherry, and characterized them with the parameter of Td-Dead and Td-iCasp9.

\section{Single-cell data analysis}

We ran different independent single-cell imaging experiments in this work, and the data from three independent experiments were shown in Supplementary Figs. 3 and 4. All the single-cell data shown in the main text are from one typical experiment. In our measurements, we first focused on $0.25 \mathrm{nM}$ inducer as this dose provided comparable population sizes of surviving and dead cells in our experimental system. We treated iCasp9 cells with $0.25 \mathrm{nM}$ inducer and tracked them using a confocal microscope for $24 \mathrm{~h}$ as apoptotic events had largely ceased by this time (Fig. 1d). Longer treatment time would result in a decreased death percentage due to the division of surviving cells (Supplementary Fig. $5 \mathrm{~b}$ and Supplementary Video 2). Then, we took $0.025,0.25$, and $2.5 \mathrm{nM}$ as representatives of the low, medium, and high concentrations, given the cell death percentages in these three concentrations were $\sim 30 \%, \sim 60 \%$, and $\sim 90 \%$, respectively (blue, green, and orange dash lines, Fig. 5a).

\section{DATA AVAILABILITY}

All data generated or analyzed during this study are included in the main text and the supplementary information files.

\section{REFERENCES}

1. Fischer U, Schulze-Osthoff K. Apoptosis-based therapies and drug targets. Cell Death Differ. 2005;12:942-61.

2. Zhou X, Di Stasi A, Brenner MK. iCaspase 9 suicide gene system. Methods Mol Biol. 2015;1317:87-105.

3. Iuliucci JD, Oliver SD, Morley S, Ward C, Ward J, Dalgarno D, et al. Intravenous safety and pharmacokinetics of a novel dimerizer drug, AP1903, in healthy volunteers. J Clin Pharmacol. 2001;41:870-9.

4. Wang $X$. The expanding role of mitochondria in apoptosis. Genes Dev. 2001;15:2922-33.

5. Xie X, Zhao X, Liu Y, Zhang J, Matusik RJ, Slawin KM, et al. Adenovirus-mediated tissue-targeted expression of a caspase-9-based artificial death switch for the treatment of prostate cancer. Cancer Res. 2001;61:6795-804.

6. Kemper K, Rodermond H, Colak S, Grandela C, Medema JP. Targeting colorectal cancer stem cells with inducible caspase-9. Apoptosis. 2012;17:528-37.

7. Nör JE, Hu Y, Song W, Spencer DM, Nuúñez G. Ablation of microvessels in vivo upon dimerization of iCaspase-9. Gene Ther. 2002;9:444-51.

8. Tey SK, Dotti G, Rooney CM, Heslop HE, Brenner MK. Inducible caspase 9 suicide gene to improve the safety of allodepleted T cells after haploidentical stem cell transplantation. Biol Blood Marrow Transplant. 2007;13:913-24.

9. Zhou X, Di Stasi A, Tey SK, Krance RA, Martinez C, Leung KS, et al. Long-term outcome after haploidentical stem cell transplant and infusion of $\mathrm{T}$ cells expressing the inducible caspase 9 safety transgene. Blood. 2014;123:3895-905.

10. Tey S-K. Adoptive T-cell therapy: adverse events and safety switches. Clin Transl Immunol. 2014;3:e17.

11. Zhang P, Raju J, Ullah MA, Au R, Varelias A, Gartlan $\mathrm{KH}_{\text {, et }}$ al. Phase I trial of inducible caspase $9 \mathrm{~T}$ cells in adult stem cell transplant demonstrates massive clonotypic proliferative potential and long-term persistence of transgenic T cells. Clin Cancer Res. 2019;25:1749-55.

12. Rossignoli F, Grisendi G, Spano C, Golinelli G, Recchia A, Rovesti G, et al. Inducible caspase9-mediated suicide gene for MSC-based cancer gene therapy. Cancer Gene Ther. 2019;26:11-16.

13. Ramos CA, Asgari Z, Liu E, Yvon E, Heslop HE, Rooney CM, et al. An inducible caspase 9 suicide gene to improve the safety of mesenchymal stromal cell therapies. Stem Cells (Dayt, Ohio). 2010;28:1107-15.

14. Wu C, Hong SG, Winkler T, Spencer DM, Jares A, Ichwan B, et al. Development of an inducible caspase- 9 safety switch for pluripotent stem cell-based therapies. Mol Ther Methods Clin Dev. 2014;1:14053.

15. Navarro SA, Carrillo E, Griñán-Lisón C, Martín A, Perán M, Marchal JA, et al. Cancer suicide gene therapy: a patent review. Expert Opin Ther Pat. 2016;26:1095-104.

16. Duarte S, Carle G, Faneca H, Lima MCP, de, Pierrefite-Carle V. Suicide gene therapy in cancer: where do we stand now? Cancer Lett. 2012;324:160-70.

17. Kreso A, O'Brien CA, Van Galen P, Gan Ol, Notta F, Brown AMK, et al. Variable clonal repopulation dynamics influence chemotherapy response in colorectal cancer. Science. 2013;339:543-8.

18. Sharma SV, Lee DY, Li B, Quinlan MP, Takahashi F, Maheswaran S, et al. A chromatin-mediated reversible drug-tolerant state in cancer. Cell Subpopul Cell. 2010;141:69-80.

19. Cohen AA, Geva-Zatorsky N, Eden E, Frenkel-Morgenstern M, Issaeva I, Sigal A, et al. Dynamic proteomics of individual cancer cells in response to a drug. Science. 2008;322:1511-6.

20. Spencer SL, Gaudet S, Albeck JG, Burke JM, Sorger PK. Non-genetic origins of cellto-cell variability in TRAIL-induced apoptosis. Nature. 2009;459:428-32.

21. Paek AL, Liu JC, Forrester WC, Lahav G, Paek AL, Liu JC, et al. Article cell-to-cell variation in p53 dynamics leads to fractional killing. Cell. 2016;165:1-12.

22. Roux J, Hafner M, Bandara S, Sims JJ, Hudson H, Chai D, et al. Fractional killing arises from cell-to-cell variability in overcoming a caspase activity threshold. Mol Syst Biol. 2015;11:803.

23. Shimbo K, Hsu GW, Nguyen $H$, Mahrus S, Trinidad JC, Burlingame AL, et al. Quantitative profiling of caspase-cleaved substrates reveals different druginduced and cell-type patterns in apoptosis. Proc Natl Acad Sci USA. 2012;109:12432-7.

24. Crawford ED, Wells JA. Caspase substrates and cellular remodeling. Annu Rev Biochem. 2011;80:1055-87.

25. Tait S, Green D. Mitochondrial regulation of cell death. Cold Spring Harb Perspect Biol. 2013;5:1-15.

26. Shiozaki EN, Chai J, Rigotti DJ, Riedl SJ, Li P, Srinivasula SM, et al. Mechanism of XIAP-mediated inhibition of caspase-9. Mol Cell. 2003;11:519-27.

27. Setiawan D, Kazaryan A, Martoprawiro MA, Filatov M. A first principles study of fluorescence quenching in rhodamine $B$ dimers: How can quenching occur in dimeric species? Phys Chem Chem Phys. 2010;12:11238-44.

28. Ogawa M, Kosaka N, Choyke PL, Kobayashi H. H-type dimer formation of fluorophores: a mechanism for activatable, in vivo optical molecular imaging. ACS Chem Biol. 2009;4:535-46.

29. Kruitwagen T, Denoth-Lippuner A, Wilkins BJ, Neumann H, Barral Y. Axial contraction and short-range compaction of chromatin synergistically promote mitotic chromosome condensation. eLife. 2015;4:1-19.

30. Rehm M, Düßmann $H$, Jänicke RU, Tavaré JM, Kögel D, Prehn JHM. Single-cell fluorescence resonance energy transfer analysis demonstrates that caspase activation during apoptosis is a rapid process: Role of caspase-3. J Biol Chem. 2002;277:24506-14.

31. Hörnle M, Peters N, Thayaparasingham B, Vörsmann H, Kashkar H, Kulms D. Caspase-3 cleaves XIAP in a positive feedback loop to sensitize melanoma cells to TRAIL-induced apoptosis. Oncogene. 2011;30:575-87.

32. Kell DB, Oliver SG How drugs get into cells: tested and testable predictions to help discriminate between transporter-mediated uptake and lipoidal bilayer diffusion. Front Pharmacol. 2014. https://doi.org/10.3389/fphar.2014.00231.

33. Banta JA, Richards CL. Quantitative epigenetics and evolution. Heredity. 2018;121:210-24.

\section{ACKNOWLEDGEMENTS}

We thank Profs. Xiaodong Wang, Jianguo Chen, and Ping Wei for kindly providing us the cells and DNA plasmids, Lucas Carey for helpful discussions, Tanqiu Liu for image analysis, Jiarong Peng and Jing Xia for data acquisition.

\section{AUTHOR CONTRIBUTIONS}

$C T$ and $Y Y$ designed the project. $Y Y$ and $Y L$ designed and performed the experiments. $Y Y, H R$, and SQ analyzed the data. $C T$ and $X Y$ supervised the whole project; $Y Y, X Y$, and $C T$ wrote the paper. 
FUNDING

This work was supported by The National Key Research and Development Program of China: 2018YFA0900700 and the National Natural Science Foundation of China (12090053 and 32088101).

\section{ETHICS STATEMENT}

Ethical approval and informed consent were not required for this study.

\section{COMPETING INTERESTS}

The authors declare no competing interests.

\section{ADDITIONAL INFORMATION}

Supplementary information The online version contains supplementary material available at https://doi.org/10.1038/s41419-021-04468-z.

Correspondence and requests for materials should be addressed to Xiaojing Yang or Chao Tang.
Reprints and permission information is available at http://www.nature.com/ reprints

Publisher's note Springer Nature remains neutral with regard to jurisdictional claims in published maps and institutional affiliations.

Open Access This article is licensed under a Creative Commons Attribution 4.0 International License, which permits use, sharing, adaptation, distribution and reproduction in any medium or format, as long as you give appropriate credit to the original author(s) and the source, provide a link to the Creative Commons license, and indicate if changes were made. The images or other third party material in this article are included in the article's Creative Commons license, unless indicated otherwise in a credit line to the material. If material is not included in the article's Creative Commons license and your intended use is not permitted by statutory regulation or exceeds the permitted use, you will need to obtain permission directly from the copyright holder. To view a copy of this license, visit http://creativecommons. org/licenses/by/4.0/.

(c) The Author(s) 202 